\title{
Increased light outcoupling efficiency in dye-doped small molecule organic light-emitting diodes with horizontally oriented emitters
}

\author{
Jörg Frischeisen, ${ }^{1 *}$ Daisuke Yokoyama, ${ }^{2,3,4 * *}$ Ayataka Endo, ${ }^{2}$ Chihaya Adachi, ${ }^{2,4,5}$ and \\ Wolfgang Brütting ${ }^{1 * * *}$
}

\footnotetext{
${ }^{1}$ Institute of Physics, University of Augsburg, Universitätsstraße 1, 86159 Augsburg, Germany

${ }^{2}$ Center for Future Chemistry, Kyushu University, 744 Motooka, Nishi, Fukuoka 819-0395, Japan

${ }^{3}$ Graduate School of Science and Engineering, Yamagata University, 4-13-16 Johnan, Yonezawa, Yamagata 992-8510, Japan

${ }^{4}$ Life Beans Kyushu, BEANS (Bio Electromechanical Autonomous Nano Systems) Laboratory, 744 Motooka, Nishi, Fukuoka 819-0395, Japan

${ }^{5}$ Center for Organic Photonics and Electronics Research (OPERA), Kyushu University, 744 Motooka, Nishi, Fukuoka 819-0395, Japan

*E-mail: Joerg.Frischeisen@physik.uni-augsburg.de

**E-mail: d_yokoyama@yz.yamagata-u.ac.jp

***E-mail: Wolfgang.Bruetting@physik.uni-augsburg.de
} 


\begin{abstract}
Small molecule organic light-emitting diodes (SM-OLEDs) are efficient large area light sources facing their market entry. However, a low light outcoupling efficiency of typically $20 \%$ still strongly limits device performance. Here, we highlight the potential of employing dye-doped emission layers with emitting molecules having horizontally oriented transition dipole moments. The effect of molecular orientation is explained by studying optical simulations that distinguish between horizontal and vertical dipole orientation. In addition, an experimental method that enables straightforward determination of dipole orientation in guest-host systems is presented and used for the analysis of two materials that are very similar except for their orientation. By measuring the external electroluminescence quantum efficiency of SM-OLEDs based on these materials, evidence is found that a mainly horizontal dipole orientation enhances light outcoupling by around 45\%. Furthermore, the effect of orientation in SMOLEDs offers many additional benefits concerning stack design and has fundamental implications for material choice.
\end{abstract}

Keywords: organic light-emitting diodes, light outcoupling, molecular orientation, optical simulation 


\section{Introduction}

Since the discovery of small molecule organic light-emitting diodes (SM-OLEDs) [1] and devices based on polymers [2] in 1987 and 1990, respectively, enormous progress has been made concerning their power conversion efficiency, lifetime and particularly electroluminescence (EL) quantum efficiency through novel materials and device architectures [3,4]. White OLEDs are now on the edge of being introduced as commercial light sources. Apart from its spectral properties the most important parameter characterising an OLED is the external EL quantum efficiency $\eta_{\mathrm{EQE}}$ that describes the ratio between the number of emitted photons and injected electrons and holes [5]:

$$
\eta_{\mathrm{EQE}}=\gamma \eta_{\mathrm{ST}} q \eta_{\mathrm{out}}
$$

Here, $\gamma$ measures the charge carrier balance, $\eta_{S T}$ is the fraction of spin-allowed excitons, $q$ represents the radiative quantum efficiency of the emitting material and $\eta_{\text {out }}$ is the outcoupling efficiency. By the use of multi-layer stacks with doped transport and additional blocking layers, the charge carrier balance can be brought close to unity. The spin-allowed exciton fraction is believed to have a value of $25 \%$ for fluorescent emitters (singlet excitons) according to quantum statistics $[6,7]$ although this value might be enhanced by triplet-triplet annihilation [8-10]. By using phosphorescent materials that harvest both singlet and triplet excitons, the factor $\eta_{\mathrm{ST}}$ can be raised to $100 \%[11,12]$.

The factors $\gamma, \eta_{\mathrm{ST}}$ and $q$ together yield the internal quantum efficiency $\eta_{\text {int. }}$. By the employment of phosphorescent emitters with high radiative quantum efficiency, $\eta_{\text {int }}$ can approach values close to one $[13,14]$. However, the most important factor still strongly limiting $\eta_{\mathrm{EQE}}$ is the outcoupling efficiency $\eta_{\mathrm{out}}$ Due to the large difference in refractive index between air $(n=1.0)$, glass $(n \approx 1.5)$ and the organic layers $(n \approx 1.7-2.0)$, a large fraction of the emitted light can not leave the device due to total internal reflection and is trapped in the substrate or as waveguide modes in the thin film structure. In addition, the excitation of surface plasmons at the interface to the metallic cathode further reduces the light extraction efficiency to values typically around 20\% [15]. By applying outcoupling enhancement structures like prisms, microlense arrays or microstructured substrates it is possible to extract a large 
fraction of substrate modes $[3,16-18]$ and bring $\eta_{\text {out }}$ up to some 30 to $40 \%$ at most, still representing a major limitation for high quantum efficiency.

Although, at present, SM-OLEDs incorporating dye-doped emitting layers are superior to their polymeric counterparts concerning efficiency and lifetime, e.g. due to the straightforward implementation of doped transport layers as well as additional blocking and injection layers in OLEDs based on small molecules leading to better charge balance factor and a well-defined recombination zone, polymer OLEDs often have the advantage of emitters being oriented parallel to the substrate plane [19-22]. Thus, the transition dipole moment that is usually oriented along the $\pi$-conjugated polymer chain is oriented in-plane. This considerably reduces coupling to unfavourable loss channels and can increase $\eta_{\text {out }}$ by a factor of approximately 1.5 compared to a random orientation of the transition dipole moments [20]. For more than 20 years, however, it has generally been believed that evaporated amorphous films in SM-OLEDs have no preferential orientation of the molecules [19]. Though there have been reports about horizontally oriented molecules in evaporated neat films [2325], only recently a non-isotropic dipole orientation in dye-doped films has been reported [25], opening up new possibilities for improvement of light outcoupling efficiency of OLEDs.

In this article we first explain the fundamental dependence of $\eta_{\text {out }}$ on the dipole orientation by studying detailed optical simulations. Next, we investigate the orientation of the transition dipole moments in two blue fluorescent dyes, 4,4'-bis[4-(diphenylamino)styryl]biphenyl (BDASBi) [26] and 4-(2,2diphenylethenyl)-N,N-bis(4-methylphenyl)benzenamine (PEBA) [27], doped into a 4,4'-bis( $N$ carbazole)-biphenyl (CBP) matrix. Angular dependent photoluminescence measurements yield strong evidence for a preferentially horizontal orientation for BDASBi and a random orientation for PEBA. Using BDASBi doped into CBP, horizontally oriented emitters are intentionally employed in dye-doped SM-OLEDs for the first time. By comparing to the PEBA reference device with random dipole orientation, strong evidence is found that the employment of oriented emitters can induce a substantial efficiency boost of almost $50 \%$ which is necessary for a widespread application of OLEDs in general lighting. 


\section{Materials, device structure and experimental methods}

Organic semiconductor films generally show optical anisotropy only if the molecular structure is anisotropic as well $[24,25]$. Because organic solids are bound only by weak intermolecular van der Waals interaction, orientation effects can occur on any underlying substrate and even in a host matrix [25]. Figure 1(a) shows the molecular structures of BDASBi and PEBA which are used as exemplary materials in this study. BDASBi is known to show a strong horizontal orientation when doped into CBP [28]. In contrast to BDASBi, which has an elongated structure, PEBA is a rather small and compact molecule. Thus, it is expected that PEBA will show much less pronounced orientation effects than BDASBi. The photoluminescence spectra of BDASBi and PEBA doped at a content of $6 \mathrm{wt} \%$ into CBP are presented in Fig. 1(b). Both spectra are very similar and have the same peak wavelength at around $470 \mathrm{~nm}$. In addition, a comparable radiative quantum efficiency $q$ of 0.65 and 0.55 was determined for 6 wt\%-BDASBi:CBP and PEBA:CBP films, respectively. Thus, both materials will experience comparable cavity effects inside an OLED device.

Figure 2 shows the basic OLED structure that is investigated in this work. The devices consist of a glass substrate covered with a patterned indium tin oxide (ITO) layer with $100 \mathrm{~nm}$ thickness, then $40 \mathrm{~nm}$ of the hole transport material $N, N^{\prime}$-diphenyl- $N, N^{\prime}$-bis(3-methylphenyl)-1,1'-biphenyl-4,4'-diamine (TPD), $10 \mathrm{~nm}$ of either BDASBi or PEBA doped into CBP (6 wt $\%$ ), $50 \mathrm{~nm}$ of the electron transport material 4,7-diphenyl-1,10-phenanthroline (Bphen) and a cathode consisting of $100 \mathrm{~nm} \mathrm{Mg:Ag} \mathrm{(10:1)}$ and a $10 \mathrm{~nm}$ thick Ag capping layer.

All organic layers were deposited in a vacuum of $<3 \times 10^{-3} \mathrm{~Pa}$ at an evaporation rate of $\sim 0.2 \mathrm{~nm} / \mathrm{s}$ except for the cathode, which was fabricated at a rate of $\sim 0.1 \mathrm{~nm} / \mathrm{s}$. All layers of the BDASBi and PEBA OLEDs except for the doped emission layer were deposited simultaneously in order to rule out differences in layer thicknesses. In addition, the substrates were rotated at equivalent positions from the axis during deposition. The active area of the OLEDs was circularly shaped with a diameter of $1 \mathrm{~mm}$.

The current density-voltage-luminance characteristics of the OLEDs were measured using a semiconductor parameter analyzer (E5273A, Agilent Co.) with an optical power meter (1930C, 
Newport Co.). The zero-degree spectra of the OLEDs were measured using a multichannel spectrometer (SD2000, Ocean Optics Co.) through an optical fibre whose end was located vertically towards the substrate of the working OLEDs. The silicon photodiode of the optical power meter was calibrated at a wavelength of $485 \mathrm{~nm}$, corresponding to the emission peak wavelength of the OLEDs. The emission spectra of the OLEDs are sufficiently narrow to neglect the spectral dependence of the response of the silicon photodiode. The photodiode captures light within a cone of approximately $56^{\circ}$. For the calculation of EQE values from the measurement, a Lambertian emission was assumed which compares reasonably well with measured and simulated angular dependent spectra.

Samples for photoluminescence (PL) and quantum efficiency measurements comprised $50 \mathrm{~nm}$ thick doped films of $6 \mathrm{wt} \%$ BDASBi or PEBA in CBP deposited on cleaned fused silica substrates. PL emission spectra were measured using a fluorescence spectrometer (FP-6500, Jasco Co.). The chosen thickness of the samples ensures that the spectra are not changed too much by waveguiding effects and that they match the emission spectra in free space. PL quantum efficiencies were determined using an absolute PL quantum yield measurement system (C9920-02, Hamamatsu Photonics K.K.).

Samples for angular dependent PL measurements consisted of $12 \mathrm{~nm}$ thick doped films of $6 \mathrm{wt} \%$ BDASBi or PEBA in CBP deposited on cleaned glass substrates and capped with a $70 \mathrm{~nm}$ thick neat CBP layer. For the measurement, a fused silica half cylinder prism was attached to the substrate side of the samples by index matching fluid and the samples were mounted on a computer controlled rotation stage. A $375 \mathrm{~nm} \mathrm{cw}$ laser diode incident under $45^{\circ}$ through the encapsulated side was used as excitation source. Since CBP has only a small absorption at this wavelength, emission from the matrix is suppressed. The angular dependent PL spectrum from 0 to $90^{\circ}$ was measured with a fibre optical spectrometer and a polarizer to distinguish between $s$ - and p-polarized emission (for details see Ref. [28]). 


\section{Optical simulation of OLEDs}

The effect of differently oriented transition dipole moments is most obvious by studying the results of an optical simulation which is based on a dipole model $[15,29,30,31]$. In this model, the dipoles are treated as damped forced harmonic oscillators embedded in a multilayer OLED. Without loss of generality, the substrate plane is assumed to be in the $x-y$-plane and the emission into the $x$-z-plane is considered, cf. Fig. 2. The simulation distinguishes three discrete dipole orientations $p_{\mathrm{x}}, p_{\mathrm{y}}$ and $p_{\mathrm{z}}$. According to the chosen coordinate system, the $p_{\mathrm{x}}$ - and $p_{\mathrm{y}}$-dipoles are oriented horizontally whereas the $p_{z}$-dipoles have a vertical orientation. The $p_{y}$-dipoles emit only $s$-polarized light whereas the $p_{x^{-}}$ and $p_{z}$-dipoles are accounting for the $p$-polarized emission.

Figure 3(a) shows the total dissipated optical power in a device with isotropic emitter orientation, where the emission spectrum is assumed to resemble the one of BDASBi:CBP (6 wt $\%)$ and the radiative quatum efficiency is set to 1 . Since CBP has higher hole than electron mobility [32] the emitter position is assumed to be at the interface to Bphen. Obviously, a large fraction of the dissipated power is coupled to two sharp waveguide modes (region 3 ) and to surface plasmons (region 4).

The contribution of each dipole orientation to the total power dissipation can be seen in Fig. 3(b), which shows a cross section of Fig. $3(a)$ at $470 \mathrm{~nm}$. The horizontally oriented $p_{x}$ - and $p_{y}$-dipoles have their major contributions in regions (1) and (2) and couple to a p-polarized and an s-polarized waveguide mode (region 3 ), respectively. By contrast, the vertically oriented $p_{z}$-dipoles show almost no emission into air or substrate but couple most of their power to surface plasmons (region 4) [33]. This behaviour can be explained by taking into account that linear dipoles radiate strongest perpendicular to their direction of oscillation [31]. Thus the vertically oriented $p_{z}$-dipoles emit mainly at large angles, i.e. high in-plane wave vectors, hence dissipating their power to an unfavourable loss channel.

The fraction of power dissipated by each dipole orientation to different optical channels is displayed in Table 1. Again, the values for the $p_{z}$-dipoles clearly show that this dipole orientation does hardly contribute at all to the desired emission into air or substrate. Consequently, the outcoupling efficiency 
can be significantly enhanced, if only horizontally oriented dipoles are present. This is apparent from the last two columns in Table 1 which show a comparison of OLEDs consisting of a completely random and a purely horizontal dipole orientation, respectively. Both the direct emission and the substrate emission are enhanced by more than $50 \%$, demonstrating the enormous potential of using horizontally oriented emitters in SM-OLEDs.

The main reason for the increased efficiency is a reduced coupling to surface plasmons. This is even more obvious by investigating the distance dependence of the emitter to the metallic mirror. Figures 3(c,d) show a variation of the electron transport layer thickness for random and horizontal dipole orientation, respectively. The oscillatory behaviour results from interference effects of the emitter with its image dipole $[31,34,35]$. The graphs clearly display the increased outcoupling efficiency for a horizontal dipole orientation. In addition, in this case the emitter may be positioned much closer to the cathode before the coupling to surface plasmons becomes the dominant loss channel. Apparently, this is an important aspect considering stack design and OLED optimization as will be discussed later.

\section{Experimental results and discussion}

\subsection{Determination of dipole orientation by photoluminescence measurements}

For a conclusive study of orientation effects in dye-doped SM-OLEDs it is necessary to use appropriate materials that differ primarily in the orientation of their transition dipole moments. The investigation of neat films by variable angle spectroscopic ellipsometry (VASE) often yields a first indication about molecular anisotropy. In this case, the optical constants determined by VASE for neat films of BDASBi indicate a strong optical anisotropy while the result for PEBA shows almost no anisotropy, however, VASE cannot yield reliable information about dye orientation in guest-host systems.

The horizontal orientation of emitting molecules as in the neat film of BDASBi can also be observed in doped films. A first demonstration of orientation in doped films was performed by edge emission measurements [25]. A more profound conclusion about the orientation in doped films can be achieved 
quantitatively from an angular dependent $p$-polarized photoluminescence measurement of a thin film and comparison with optical simulations [28]. In this experiment a stack without metallic layers is used so that the emission from vertically oriented $p_{z}$-dipoles is directly measurable and information about their existence and even the exact ratio between horizontal and vertical dipoles can be obtained. The stack under investigation comprises a $12 \mathrm{~nm}$ thick layer of CBP containing $6 \mathrm{wt} \%$ BDASBi or PEBA, respectively, capped with a $70 \mathrm{~nm}$ thick neat CBP layer. Figures $4(\mathrm{a}, \mathrm{b})$ display the $p$-polarized simulation results of the BDASBi sample for a completely random and a purely horizontal dipole orientation, respectively. By comparing the measured spectra shown in Fig. 4(c) with the simulations, it is apparent that the transition dipoles in a BDASBi:CBP layer are primarily horizontally oriented. A precise analysis of the dipole orientation is performed by comparing the cross sections of measurement and simulations, which is displayed in Fig. 5. In addition, the cross section of a stack containing PEBA instead of BDASBi with otherwise identical structure is shown. Comparison of the measured cross section for PEBA with the simulated curves yields excellent agreement assuming a random dipole orientation. Thus, PEBA doped into CBP shows no preferred molecular orientation and the dipole orientation is completely random, i.e. on average $2 / 3$ of the dipoles are horizontally and $1 / 3$ vertically oriented. On the contrary, the cross section of the sample containing BDASBi shows a clear tendency towards horizontal orientation. By weighting the simulations for random and horizontal dipole orientations with 0.27 and 0.73 , respectively, very good agreement between the simulated and measured curve can be obtained. These values are in excellent agreement with Ref. [28]. Consequently, it follows that the total ratio between $p_{z}$ and $p_{x}$ is $0.27: 1$. Since the horizontal $p_{x}-$ and $p_{\mathrm{y}}$-dipoles are uniformly distributed in the substrate plane, the overall fraction of vertical $\left(p_{z}\right)$ dipoles is $0.27 / 2.27=12 \%$ and the fraction of horizontal $\left(p_{x}, p_{y}\right)$ dipoles is $2 / 2.27=88 \%$.

We note that quantitative information about emitter orientation can also be obtained by fluorescence lifetime measurements, as reported in Ref. [31]. This requires the preparation of a series of samples with systematically varying distance between the emission zone and a metal layer. Though being more elaborate, this method has the benefit of independently yielding the value of the radiative 
quantum efficiency of the emitter, too, as will be published separately for a red phosphorescent OLED [36].

\subsection{Quantum efficiency of dye-doped OLEDs}

As a result of the previous analysis, BDASBi and PEBA are ideal candidates for studying the effect of molecular orientation in actual OLED devices. Both materials were incorporated in a rather simple stack in order to rule out differences in device fabrication. The same stack layout as already discussed in the simulations is employed, cf. Fig. 2. Neglecting any effects of orientation, it is expected that the OLED based on BDASBi has a 1.16 times larger external quantum efficiency than the OLED with PEBA because of the difference in radiative quantum efficiency $q$. The simulations can also be used to predict the influence of orientation which should additionally boost the efficiency of the OLED with BDASBi. Assuming $88 \%$ horizontal orientation from the analysis above, it is expected that the OLED based on BDASBi gains an extra enhancement of outcoupling efficiency and therewith external quantum efficiency of $37 \%$. If BDASBi were perfectly oriented horizontally, this enhancement would even amount to $59 \%$.

For a conclusive determination of $\eta_{\mathrm{out}}$ from a measurement of $\eta_{\mathrm{EQE}}$ it is critical that all other factors in equation (1) are comparable. First, the spin-allowed exciton fraction $\eta_{\mathrm{ST}}$ is generally accepted to have a value of $25 \%$ in SM-OLEDs based on fluorescent emitters [6,7]. Second, the radiative quantum efficiency $q$ was determined to be 0.65 for the 6 wt\%-BDASBi:CBP and 0.55 for PEBA:CBP layers. This difference must be included in optical simulations due to different cavity enhancement $[15,37,38]$. Finally, the charge balance factor $\gamma$ is mainly governed by the transport layers TPD and Bphen. Since both OLEDs comprise the same structure it can be assumed that $\gamma$ is rather similar in both devices. Although charge trapping effects in the doped CBP matrix can not be fully ruled out due to slightly different energy of the lowest unoccupied molecular orbitals (LUMO) of BDASBi and PEBA [39], the almost identical current-voltage characteristics (see below) give strong evidence that minor differences in charge carrier transport and recombination in both devices do not play a dominant role. In addition, it is necessary to have information concerning the emitter position and therewith the distance to the 
cathode as this has an important influence for the simulated outcoupling efficiency as was demonstrated in Figs. 3(c,d). Since both BDASBi and PEBA contain triphenylamine substituents it is expected that they have a higher hole than electron mobility. CBP also shows a slightly larger hole than electron mobility, therefore it is reasonable to assume that the emission takes place close to the interface between the emitting layer and Bphen. However, the assumptions concerning charge balance and emitter position are further verified by investigating the current-voltage characteristics and the spectrum at different current densities (Fig. 6).

Figure 6(a) shows the current-voltage characteristics for two OLEDs based on BDASBi and PEBA as emitters, respectively. Since the OLEDs only differ in their dopant, the measured current density is nearly the same for both devices. This strengthens the assumption of comparable charge balance. The zero-degree spectra of both OLEDs are plotted in Fig. 6(b) for different current densities. The spectra are almost independent of current density, confirming that the emitter position does not depend significantly on current density as otherwise the spectra would shift due to changing cavity effects [34]. In addition, Fig. 6(b) illustrates again that the spectra of OLEDs fabricated with both materials are well comparable.

The external quantum efficiency is shown in Fig. 6(c). Apparently, $\eta_{\mathrm{EQE}}$ of the OLED based on BDASBi is significantly larger than only a factor of 1.16 which would be expected from the difference in radiative quantum efficiency. An average in the range between 0.1 and $10 \mathrm{~mA} / \mathrm{cm}^{2}$ yields an $\eta_{\mathrm{EQE}}$ of $2.7 \%$ for the BDASBi-stack and $1.6 \%$ for the device based on PEBA. It should be noted that the absolute values of $\eta_{\mathrm{EQE}}$ for both devices are smaller than expected from simulations of the outcoupling efficiency yielding $3.9 \%$ for random orientation and $5.9 \%$ for perfectly parallel orientation of BDASBi molecules and 3.3\% for randomly orientated PEBA molecules, respectively (see Supplementary Information S5). This is probably due to imperfect charge balance owing to the fact that no doped transport layers and selective carrier blocking layers were used in our simple OLED stack. However, since both types of OLEDs utilize identical HTL and ETL (fabricated in the same evaporation run) as well as the same matrix for the EML, we believe that both devices have quite comparable factor $\gamma$ in Eq. (1). In addition we want to emphasize that we are mainly interested in the relative increase of 
outcoupling efficiency. Thus, any effects that reduce the efficiency and occur in both types of OLEDs cancel out.

Taking the difference in radiative quantum efficiency into account, it follows that the OLED based on BDASBi exhibits an increase of outcoupling efficiency by $45 \%$ due to the strongly horizontally oriented dipoles. This is even slightly higher than the value expected from the analysis of dipole orientation in both materials, but it validates the basic result of mainly horizontally oriented dipoles in BDASBi obtained by ellipsometry and the angular dependent photoluminescence measurement. Even allowing for different emitter positions in the simulation of both devices, the outcoupling and external quantum efficiency of the OLED based on BDASBi is significantly larger. Considering that the orientation of BDASBi is not yet perfectly horizontal, this manifests the enormous efficiency enhancement that can be obtained by incorporating oriented emitters into SM-OLED devices.

\subsection{Discussion}

The well-known concept of non-isotropic emitter orientation, presented here for the first time in dyedoped SM-OLEDs, to achieve enhanced light outcoupling, is of course not limited to fluorescent materials only, but can also be applied to phosphorescent dyes [36]. Since the latter are usually rather bulky metal-organic complexes, achieving horizontally oriented emitter molecules will be more challenging than with rod-like linear molecules used here. Nevertheless, tailoring phosphorescent materials with respect to this feature, e.g. by asymmetric organic ligands, might allow for a substantial increase in external quantum efficiency from about $20 \%$ with random emitter orientation to more than $30 \%$ in the case of flat lying dipoles - without any additional outcoupling structure.

Besides the enormous boost in outcoupling and external EL quantum efficiency, the use of oriented emitters in SM-OLEDs offers a series of additional benefits. First, the emitter position may move closer to the cathode without loosing too much energy to surface plasmons. Thus, the emitter can be placed at a position where it receives optimal interference and cavity enhancement. Additionally, with horizontally oriented emitters both the first and second cavity maxima are comparable in outcoupling efficiency, which offers more freedom in stack design. The freedom to place the emitter close to the 
cathode is especially important in white OLEDs and stacked OLEDs that comprise several emitting layers [40-42]. It should be noted that the advantage of horizontally oriented dipoles disappears, when high index glass substrates $(n \approx 1.8)$ and thick electron transport layers are used in order to suppress coupling to waveguide modes and surface plasmons $[3,4,43]$. However, using substrates with such a high refractive index probably is too expensive for an application in large area OLED light sources. Furthermore, it is essential to know the correct dipole orientation in any analysis of measured $\eta_{\mathrm{EQE}}$ values, since the outcoupling efficiency can change by more than a factor of 1.5 . If the emitter orientation is not taken into account properly, this implies equivalently large errors in the determination of the internal quantum efficiency [36] and, e.g., the spin-allowed exciton fraction $[21,38]$. Considering material choice it must be taken into account that the effect of horizontally oriented emitter molecules can to some extend compensate for a lower radiative quantum efficiency as compared to an unoriented material. For example, the search for the longed-for stable deep-blue phosphorescent emitter could take a turn: The general difference of a factor of 4 in the external quantum efficiency between phosphorescent and fluorescent emitters reduces to only about 2.5 if the fluorescent emitter is horizontally oriented. Thus, present stability problems with blue phosphorescent emitters may be avoided without sacrificing too much in efficiency.

It is further noteworthy that oriented molecules in neat and doped films also play an important role in related disciplines. Because alignment enlarges the overlap of molecular orbitals between adjacent molecules, non-isotropic orientation can contribute to an improvement of the charge transport characteristics not only in OLEDs $[44,45]$ but also in organic photovoltaic cells (OPVCs) and organic field-effect transistors (OFETs). Recently, small molecule OPVCs using an amorphous material with a high carrier mobility were reported [46], where the molecule has an anisotropic planar structure. We anticipate that horizontally oriented molecules with an anisotropic structure strongly improve device performance of OPVCs due to both increased absorption and enhanced charge transport. Similarly, it is well known that OFETs profit from an enhanced charge transport resulting from oriented molecules. Especially the development of electrically pumped organic lasers based on light-emitting OFETs could benefit from oriented molecules with improved transport and light outcoupling [47]. 


\section{Conclusions and outlook}

In conclusion, an SM-OLED employing intentionally horizontally oriented emitter molecules was presented and compared to a reference device with random emitter orientation. The SM-OLED incorporating oriented emitters exhibits an increase in light-outcoupling and external quantum efficiency of about $45 \%$. Certainly, horizontally oriented phosphorescent emitters would represent the ultimate material of choice for SM-OLEDs. Our presented technique of determining the orientation in dye-doped films by a simple angular dependent photoluminescence measurement offers a straightforward method to investigate numerous guest-host combinations and newly developed materials as well as fabrication procedures that influence molecular orientation. It should be emphasized that using horizontally oriented emitters fabricated in a common evaporation process does not change production costs at all. On the contrary, comparably efficient outcoupling enhancement techniques like scattering at Bragg gratings [48] or photonic crystal structures [49] significantly complicate the fabrication process and raise the costs accordingly. 


\section{Supplementary Information}

Further information about the material properties, the optical power dissipation spectra for individual dipole orientations, the determination of orientation in neat films by VASE, the determination of dipole orientation for PEBA doped into CBP, the influence of emitter position on EQE, the influence of angular dependent emission characteristics on the EQE measurement as well as all optical constants used in the simulation can be found in the Supplementary Information.

\section{Acknowledgements}

We acknowledge financial support by the Elite Network of Bavaria through the international graduate school "Materials Science of Complex Interfaces" and by the Japan Society for the Promotion of Science (JSPS). This work was partly supported by Promotion of Environmental Improvement for Independence of Young Researchers Program of Ministry of Education, Culture, Sports, Science and Technology, Japan. We thank Prof. Junji Kido for using the ultraviolet photoelectron spectroscopy setup in his lab at Yamagata University, Japan. We also thank the Research Center for Computational Science (RCCS), Okazaki National Research Institutes, for the use of a SGI Altix4700 computer. 


\section{References}

1. C.W. Tang, S.A. VanSlyke, Organic electroluminescent diodes, Appl. Phys. Lett. 51 (1987) 913-915.

2. J.H. Burroughes, D.D.C. Bradley, A.R. Brown, R.N. Marks, K. Mackay, R.H. Friend, P.L. Burns, A.B. Holmes, Light-emitting diodes based on conjugated polymers, Nature 347 (1990) 539-541.

3. S. Reineke, F. Lindner, G. Schwartz, N. Seidler, K. Walzer, B. Lüssem, K. Leo, White organic light-emitting diodes with fluorescent tube efficiency, Nature 459 (2009) 234-239.

4. S. Mladenovski, K. Neyts, D. Pavicic, A. Werner, C. Rothe, Exceptionally efficient organic light emitting devices using high refractive index substrates, Opt. Express 17 (2009) 7562-7570.

5. T. Tsutsui, E. Aminaka, C.P. Lin, D.-U. Kim, Extended molecular design concept of molecular materials for electroluminescence: sublimed-dye films, molecularly doped polymers and polymers with chromophores, Phil. Trans. R. Soc. Lond. A 355 (1997) 801-814.

6. M.A. Baldo, D.F. O'Brien, M.E. Thompson, S.R. Forrest, Excitonic singlet-triplet ratio in a semiconducting organic thin film, Phys. Rev. B. 60 (1999) 14422-14428.

7. M. Segal, M.A. Baldo, R.J. Holmes, S.R. Forrest, Z.G. Soos, Excitonic singlet-triplet ratios in molecular and polymeric organic materials, Phys. Rev. B 68 (2003) 075211.

8. M. Pope, C.E. Swenberg, Electronic Processes in Organic Crystals and Polymers, Oxford Univ. Press $\left(2^{\text {nd }}\right.$ Ed., 1999).

9. D.Y. Kondakov, T.D. Pawlik, T.K. Hatwar, J.P. Spindler, Triplet annihilation exceeding spin statistical limit in highly efficient fluorescent organic light-emitting diodes, J. Appl. Phys. 106 (2009) 124510.

10. Y. Luo, H. Aziz, Correlation between triplet-triplet annihilation and electroluminescence efficiency in doped fluorescent organic light-emitting devices, Adv. Funct. Mater. 20 (2010) 1285.

11. M.A. Baldo, D.F. O'Brien, Y. You, A. Shoustikov, S. Sibley, M.E. Thompson, S.R. Forrest, Highly efficient phosphorescent emission from organic electroluminescent devices, Nature 395 (1998) 151-154.

12. J.S. Wilson, A.S. Dhoot, A.J.A.B. Seeley, M.S. Khan, A. Köhler, R.H. Friend, Spin-dependent exciton formation in $\pi$-conjugated compounds, Nature 413 (2001) 828-831.

13. C. Adachi, M.A. Baldo, M.E. Thompson, S.R. Forrest, Nearly $100 \%$ internal phosphorescence efficiency in an organic light emitting device, J. Appl. Phys. 90 (2001) 5048-5051.

14. E.L. Williams, K. Haavisto, J. Li, G.E. Jabbour, Excimer-based white phosphorescent organic light emitting diodes with nearly $100 \%$ internal quantum efficiency, Adv. Mater. 19 (2007) 197-202. 
15. S. Nowy, B.C. Krummacher, J. Frischeisen, N.A. Reinke, W. Brütting, Light extraction and optical loss mechanisms in organic light-emitting diodes: Influence of the emitter quantum efficiency, J. Appl. Phys. $104(2008) 123109$.

16. W.H. Koo, S.M. Jeong, F. Araoka, K. Ishikawa, S. Nishimura, T. Toyooka, H. Takezoe, Light extraction from organic light-emitting diodes enhanced by spontaneously formed buckles, Nature Photon. 4 (2010) 222-226.

17. Y. Sun, S.R. Forrest, Enhanced light out-coupling of organic light-emitting devices using embedded lowindex grids, Nature Photon. 2 (2008) 483.

18. H.-Y. Lin, J.-H. Lee, M.-K. Wei, C.-L. Dai, C.-F. Wu, Y.-H. Ho, H.-Y. Lin, T.-C. Wu, Improvement of the outcoupling efficiency of an organic light-emitting device by attaching microstructured films, Opt. Commun. 275 (2007) 464-469.

19. J.M. Ziebarth, M.D. McGehee, A theoretical and experimental investigation of light extraction from polymer light-emitting diodes, J. Appl. Phys. 97 (2005) 064502.

20. J.-S. Kim, P.K.H. Ho, N.C. Greenham, R.H. Friend, Electroluminescence emission pattern of organic lightemitting diodes: Implications for device efficiency calculations, J. Appl. Phys. 88 (2000) 1073-1081.

21. A. Köhler, J. Wilson, Phosphorescence and spin-dependent exciton formation in conjugated polymers, Org. Electron. 4 (2003) 179-189.

22. S.L.M. van Mensfoort, M. Carvelli, M. Megens, D. Wehenkel, M. Bartyzel, H. Greiner, R.A.J. Janssen, R. Coehoorn, Measuring the light emission profile in organic light-emitting diodes with nanometre spatial resolution, Nature Photon. 4 (2010) 329-335.

23. H.-W. Lin, C.-L. Lin, H.-H. Chang, Y.-T. Lin, C.-C. Wu, Y.-M. Chen, R.-T. Chen, Y.-Y. Chien, K.-T. Wong, Anisotropic optical properties and molecular orientation in vacuum-deposited ter(9,9-diarylfluorene)s thin films using spectroscopic ellipsometry, J. Appl. Phys. 95 (2004) 881-886.

24. D. Yokoyama, A. Sakaguchi, M. Suzuki, C. Adachi, Horizontal molecular orientation in vacuum-deposited organic amorphous films of hole and electron transport materials, Appl. Phys. Lett. 93 (2008) 173302.

25. D. Yokoyama, A. Sakaguchi, M. Suzuki, C. Adachi, Horizontal orientation of linear-shaped organic molecules having bulky substituents in neat and doped vacuum-deposited amorphous films, Org. Electron. 10 (2009) 127-137. 
26. H.S. Bang, S.Y. Seo, D.C. Choo, T.W. Kim, S.J. Lee, J.H. Seo, Y.K. Kim, C. Chu, J. Ha, Effect of doped emitting layer on electrical and optical properties in blue organic light-emitting devices, Thin Solid Films 517 (2009) 5314-5317.

27. T. Niimi, M. Umeda, Electron transfer between a photoexcited azo pigment particle and an electron donor molecule in a solid system, J. Phys. Chem. B 106 (2002) 3657-3661.

28. J. Frischeisen, D. Yokoyama, C. Adachi, W. Brütting, Determination of molecular dipole orientation in doped fluorescent organic thin films by photoluminescence measurements, Appl. Phys. Lett. 96 (2010) 073302.

29. R.R. Chance, A. Prock, R. Silbey, Lifetime of an emitting molecule near a partially reflecting surface, J. Chem. Phys. 60 (1974) 2744-2748.

30. W.L. Barnes, Topical review Fluorescence near interfaces: the role of photonic mode density, J. Mod. Opt. 45 (1998) 661-699.

31. S.H. Garrett, J.A.E. Wasey, W.L. Barnes, Determining the orientation of the emissive dipole moment associated with dye molecules in microcavity structures, J. Mod. Opt. 15 (2004) 2287-2295.

32. H. Matsushima, S. Naka, H. Okada, H. Onnagawa, Organic electrophosphorescent devices with mixed hole transport material as emission layer, Curr. Appl. Phys. 5 (2005) 305-308.

33. W.H. Weber, C.F. Eagen, Energy transfer from an excited dye molecule to the surface plasmons of an adjacent metal, Opt. Lett. 4 (1979) 236-238.

34. H. Becker, S.E. Burns, R.H. Friend, Effect of metal films on the photoluminescence and electroluminescence of conjugated polymers, Phys. Rev. B 56 (1997) 1893-1905.

35. Y. Cao, I.D. Parker, G. Yu, C. Zhang, A.J. Heeger, Improved quantum efficiency for electroluminescence in semiconducting polymers, Nature 397 (1999) 414-417.

36. M. Flämmich, J. Frischeisen, D.S. Setz, D. Michaelis , B.C. Krummacher , T.D. Schmidt, W. Brütting , N. Danz, to be published

37. B.C. Krummacher, S. Nowy, J. Frischeisen, M. Klein, W. Brütting, Efficiency analysis of organic lightemitting diodes based on optical simulation, Org. Electron. 10 (2009) 478-485.

38. J.A.E. Wasey, A. Safonov, I.D.W Samuel, W.L. Barnes, Efficiency of radiative emission from thin films of a light-emitting conjugated polymer, Phys. Rev. B. 64 (2001) 205201.

39. HOMO levels of BDASBi and PEBA were determined by atmospheric ultraviolet photoelectron spectroscopy (Riken Keiki, AC-3) using vacuum-deposited films. Both materials have a HOMO of $5.6 \mathrm{eV}$. 
In addition, a HOMO level of $6.0 \mathrm{eV}$ is reported for CBP [N. Matsumoto, et al., J. Phys. Chem. C 112 (2008) 7735-7741]. LUMO levels were calculated from the HOMO levels and the low energy edges of the absorption spectra of neat BDASBi, PEBA and CBP films on fused silica. The values are $2.9 \mathrm{eV}$ for BDASBi, $2.7 \mathrm{eV}$ for PEBA and $2.6 \mathrm{eV}$ for CBP. Hence, it cannot be ruled out completely that electron trapping plays a slightly more important role in the BDASBi devices. However, strong trapping effects would probably cause significant differences between BDASBi and PEBA OLEDs in the current-voltage characteristics, which is not observed in the measurement, cf. Fig. 6(a). We therefore conclude that an increase of outcoupling efficiency due to molecular orientation is the key factor for the higher EQE of BDASBi OLEDs.

40. T. Tsutsui, M. Terai, Electric field-assisted bipolar charge spouting in organic thin-film diodes, Appl. Phys. Lett. 84 (2004) 440-442.

41. L.S. Liao, K.P. Klubek, C.W. Tang, High-efficiency tandem organic light-emitting diodes, Appl. Phys. Lett. $84(2004)$ 167-169.

42. F. Guo, D. Ma, White organic light-emitting diodes based on tandem structures, Appl. Phys. Lett. 87 (2005) 173510.

43. G. Gaertner, H. Greiner, Light extraction from OLEDs with (high) index matched glass substrates, Proc. of SPIE 6999 (2008) 69992T-1-12.

44. D. Yokoyama, A. Sakaguchi, M. Suzuki, C. Adachi, Enhancement of electron transport by horizontal molecular orientation of oxadiazole planar molecules in organic amorphous films, Appl. Phys. Lett. 95 (2009) 243303.

45. D. Yokoyama, Y. Setoguchi, A. Sakaguchi, M. Suzuki, C. Adachi, Orientation control of linear-shaped molecules in vacuum-deposited organic amorphous films and its effect on carrier mobilities, Adv. Funct. Mater. 20 (2010) 386-391.

46. H. Kageyama, H. Ohishi, M. Tanaka, Y. Ohmori, Y. Shirota, High performance organic photovoltaic devices using amorphous molecular materials with high charge-carrier drift mobilities, Appl. Phys. Lett. 94 (2009) 063304.

47. H.-W. Lin, C.-L. Lin, C.-C. Wu, T.-C. Chao, K.-T. Wong, Influences of molecular orientations on stimulated emission characteristics of oligofluorene films, Org. Electron. 8 (2007) 189-197.

48. J.M. Ziebarth, A.K. Saafir, S. Fan, M.D. McGehee, Extracting light from polymer light-emitting diodes using stamped bragg gratings, Adv. Funct. Mater. 14 (2004) 451-456. 
49. Y.R. Do, Y.-C. Kim, Y.-W. Song, Y.-H. Lee, Enhanced light extraction efficiency from organic light emitting diodes by insertion of a two-dimensional photonic crystal structure, J. Appl. Phys. 96 (2004) 7629-7636. 


\section{Figures}

Figure 1

(a)
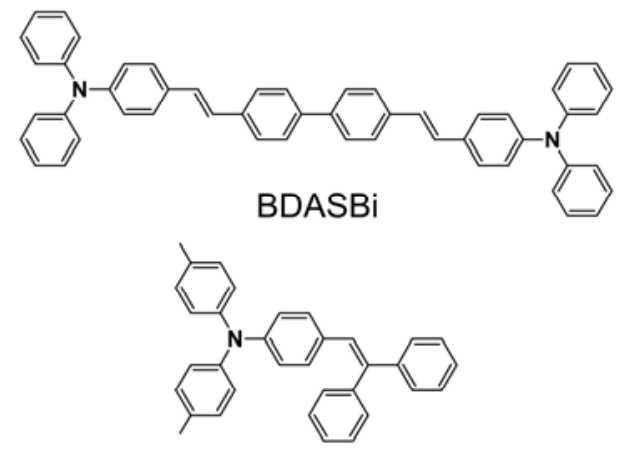

PEBA

(b)

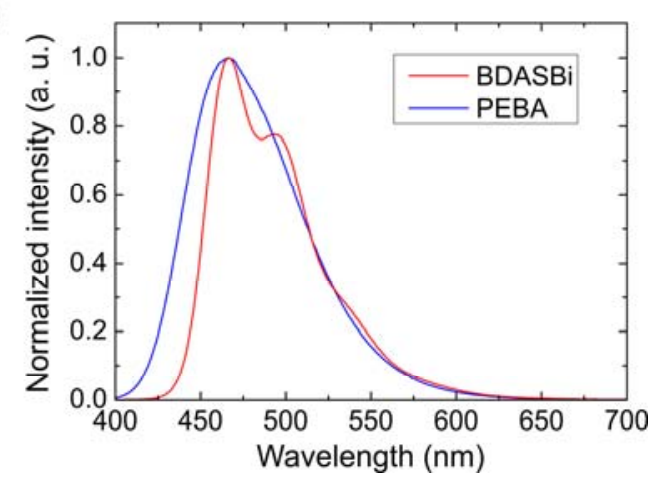

Fig. 1. (a) Molecular structures of BDASBi and PEBA. (b) Photoluminescence emission spectra of $50 \mathrm{~nm}$ films of BDASBi and PEBA doped into CBP (6 wt\%) measured perpendicular to the silica glass substrate. 
Figure 2

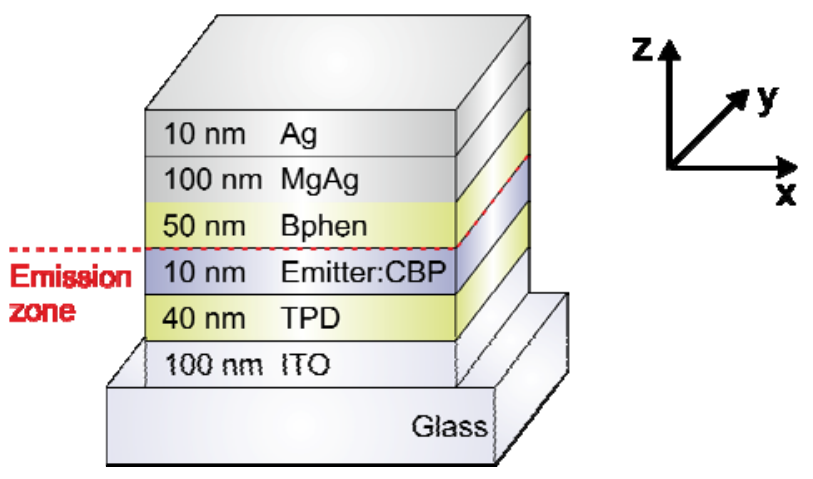

Fig. 2. Schematic diagram of the OLED structure and definition of the coordinate system and dipole orientations used in the simulation. 
Figure 3
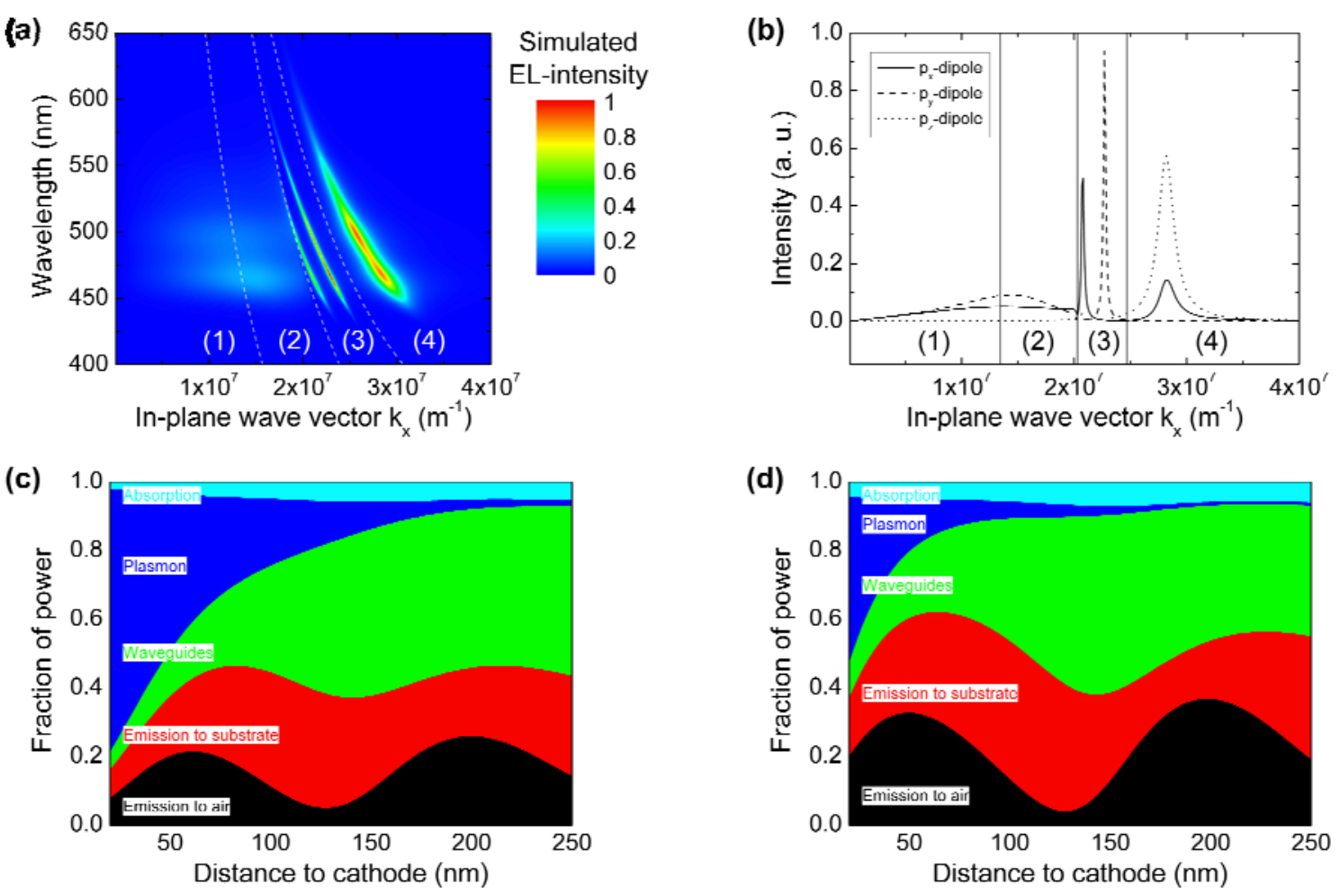

Fig. 3. (a) Simulation of the total optical power dissipation in an organic light-emitting diode as shown in Fig. 2 assuming isotropic emitter orientation with an emission spectrum resembling that of BDASBi:CBP (6 wt\%) and a radiative quantum efficiency $q=1$. Red areas indicate high amount of dissipated power. The lines divide the graph into four regions: emission into air (1), emission into the glass substrate (2), waveguide modes (3) and coupling to surface plasmons (4). (b) Cross section of (a) at a wavelength of $470 \mathrm{~nm}$. The dissipated power is split into the corresponding fractions of each dipole. The vertical lines divide the graph into four regions similar to (a). (c,d) Dissipated power in dependence of the distance to the cathode for (c) random dipole orientation and (d) horizontal dipole orientation. 
Figure 4
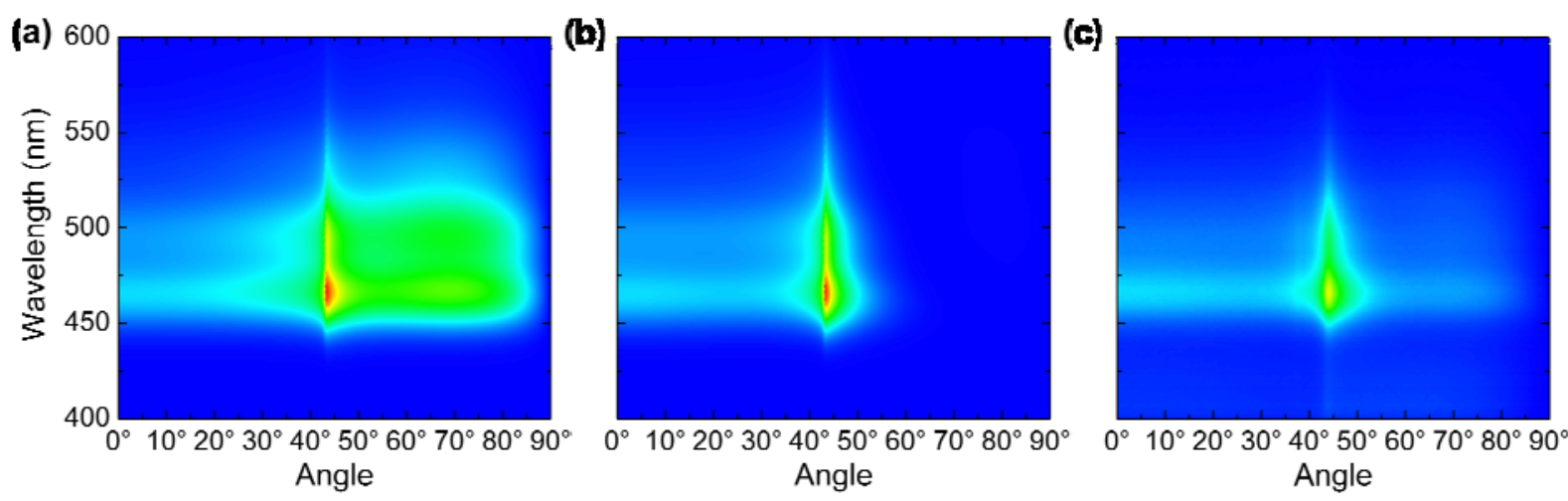

PL-intensity

(a. u.)

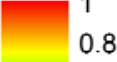

0.8

0.6

0.4

0.2

Fig. 4. Simulation and measurement of the angular dependent $p$-polarized emission spectrum of a $12 \mathrm{~nm}$ film of BDASBi:CBP (6 wt\%) on a glass substrate capped with $70 \mathrm{~nm}$ of neat CBP. Simulation assuming (a) a random dipole orientation, (b) a purely horizontal dipole orientation and (c) measured emission spectrum. The measured emission intensity is scaled so that the emission at small angles corresponds to the simulations. 
Figure 5

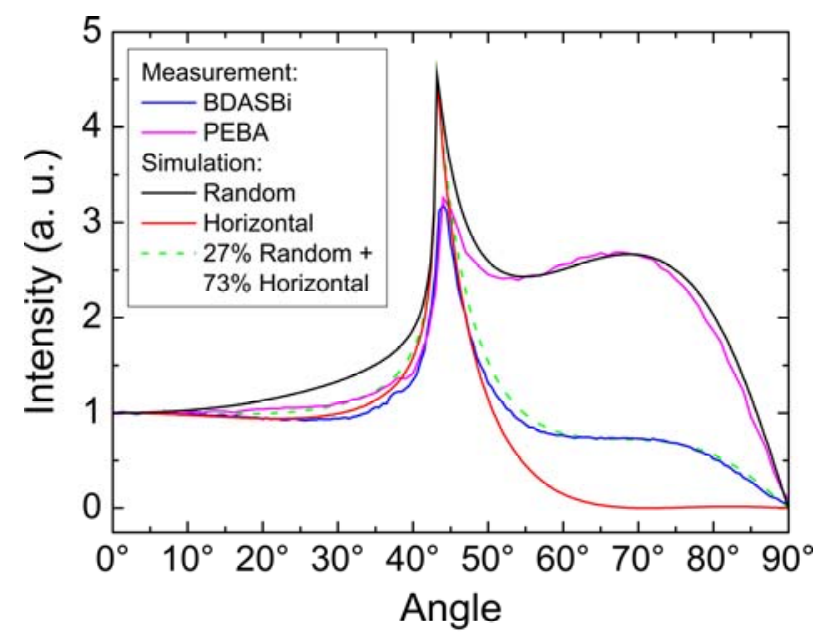

Fig. 5. Angular dependent cross section at $470 \mathrm{~nm}$ of the simulated and measured emission spectra shown in Fig. 4 and of a measurement with a sample containing PEBA instead of BDASBi. All cross sections are normalized to the emission at small angles because the emission in this range occurs exclusively from the horizontal $p_{\mathrm{x}}$-dipoles. On the other hand, the emission at angles around $70^{\circ}$ is dominated by the $p_{z}$-dipoles. The dashed line represents an average of random and horizontal dipole orientation with a relative weight of 0.27 and 0.73 , respectively. 
Figure 6
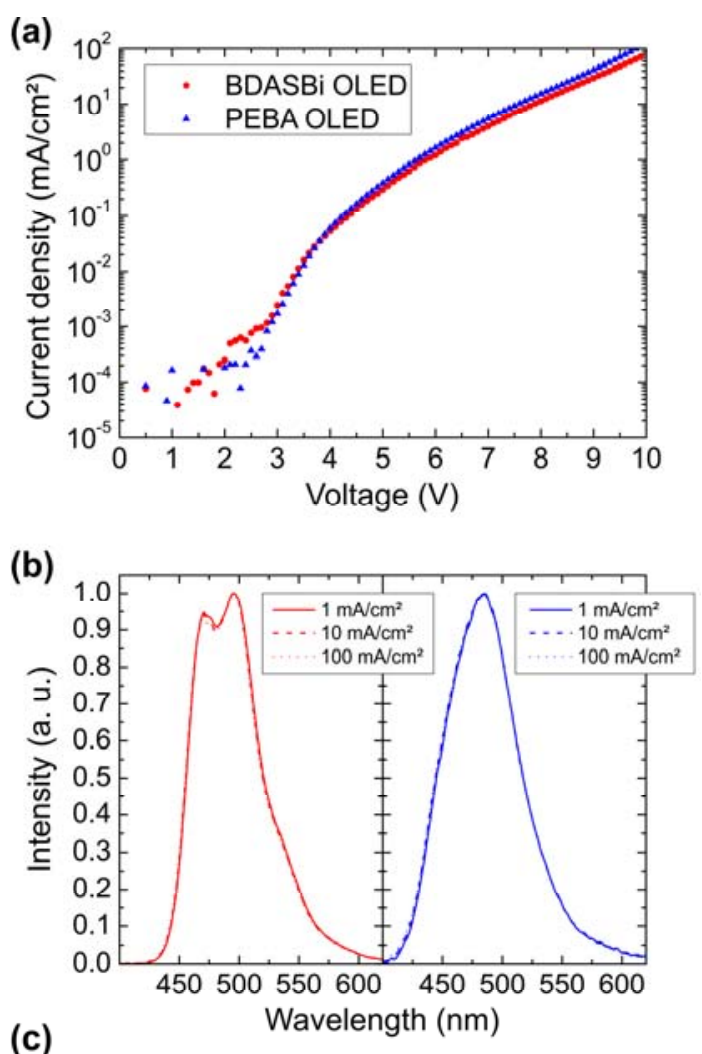

(c)

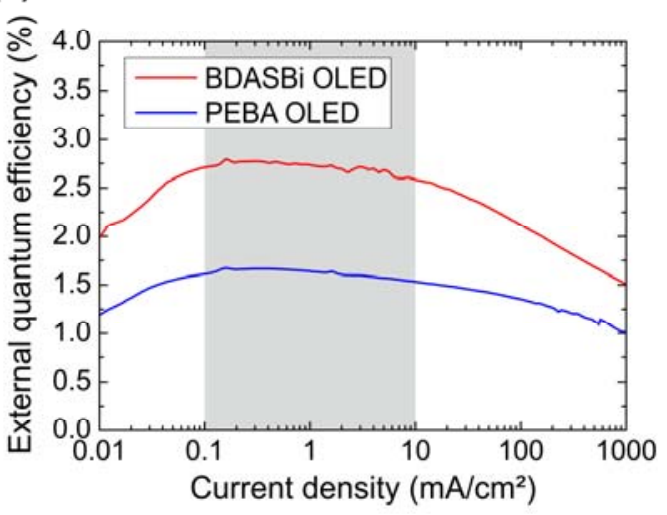

Fig. 6. (a) Measured current-voltage characteristics for OLEDs comprising either BDASBi:CBP or PEBA:CBP as an emitting layer. (b) Measured zero-degree emission spectra at different current densities. (c) Experimentally determined external EL quantum efficiencies depending on current density. The EQE values are averaged in the range between 0.1 and $10 \mathrm{~mA} / \mathrm{cm}^{2}$ because the measured curves show a plateau in this region. For smaller current densities there is probably no stable charge balance, while quenching processes occur for higher current densities. 


\section{J. Frischeisen et al.}

\section{Tables}

Table 1

\begin{tabular}{|c|c|c|c|c|c|}
\hline & $p_{\mathrm{x}}$-dipole & $p_{\mathrm{y}}$-dipole & $p_{\mathrm{z}}$-dipole & $\begin{array}{c}\text { Random } \\
\left(p_{\mathrm{x}} / p_{\mathrm{y}} / p_{\mathrm{z}}\right)\end{array}$ & $\begin{array}{c}\text { Horizontal } \\
\left(p_{\mathrm{x}} / p_{\mathrm{y}}\right)\end{array}$ \\
\hline Direct emission to air & $28.7 \%$ & $36.5 \%$ & $0.1 \%$ & $20.0 \%$ & $32.6 \%$ \\
\hline Substrate emission & $25.1 \%$ & $30.2 \%$ & $0.9 \%$ & $17.3 \%$ & $27.6 \%$ \\
\hline Waveguides & $11.5 \%$ & $27.3 \%$ & $3.4 \%$ & $13.3 \%$ & $19.4 \%$ \\
\hline Plasmons & $29.8 \%$ & $0.7 \%$ & $94.3 \%$ & $45.7 \%$ & $15.3 \%$ \\
\hline
\end{tabular}

Table 1. Dissipated power coupled into different optical channels depending on the orientation of the transition dipole moment. Simulations are based on an OLED as shown in Fig. 2 with BDASBi:CBP (6 wt\%) as an emitting layer. The residual percentage to $100 \%$ is lost due to absorption in ITO and the organic layers and at the cathode. For the last two columns it should be noted that these numbers are not simply the average of the corresponding values of each dipole. Instead of that, a weighting according to cavity effects has to be considered. 